OPEN ACCESS

Edited by:

Sheng Guo,

Chalmers University of Technology,

Sweden

Reviewed by:

Demircan Canadinc,

Koç University, Turkey

Yiping $L u$,

Dalian University of Technology (DUT),

China

*Correspondence:

Zhiming Li

zhiming.li@mpie.de

Specialty section:

This article was submitted to

Structural Materials,

a section of the journal

Frontiers in Materials

Received: 27 March 2018

Accepted: 11 May 2018

Published: 30 May 2018

Citation:

Basu S, Li Z, Pradeep KG and Raabe D (2018) Strain Rate Sensitivity of a TRIP-Assisted Dual-Phase

High-Entropy Alloy. Front. Mater. 5:30.

doi: 10.3389/fmats.2018.00030

\section{Strain Rate Sensitivity of a TRIP-Assisted Dual-Phase High-Entropy Alloy}

\author{
Silva Basu ${ }^{1,2}$, Zhiming Li ${ }^{1 *}$, K. G. Pradeep ${ }^{1,3}$ and Dierk Raabe ${ }^{1}$ \\ ${ }^{1}$ Max-Planck-Institut für Eisenforschung, Düsseldorf, Germany, ${ }^{2}$ Materials Science Centre, Indian Institute of Technology \\ Kharagpur, Kharagpur, India, ${ }^{3}$ Materials Chemistry, RWTH Aachen University, Aachen, Germany
}

Dual-phase high-entropy alloys (DP-HEAs) with transformation induced plasticity (TRIP) have an excellent strength-ductility combination. To reveal their strain-rate sensitivity and hence further understand the corresponding deformation mechanisms, we investigated the tensile behavior and microstructural evolution of a typical TRIP-DP-HEA $\left(\mathrm{Fe}_{50} \mathrm{Mn}_{30} \mathrm{Co}_{10} \mathrm{Cr}_{10}\right.$, at. \%) under different strain rates (i.e., $5 \times 10^{-3} \mathrm{~s}^{-1}, 1 \times 10^{-3}$ $\mathrm{s}^{-1}, 5 \times 10^{-4} \mathrm{~s}^{-1}$ and $1 \times 10^{-4} \mathrm{~s}^{-1}$ ) at room temperature. The strain rate range was confined to this regime in order to apply the digital image correlation technique for probing the local strain evolution during tensile deformation at high resolution and to correlate it to the microstructure evolution. Grain size effects of the face-centered cubic (FCC) matrix and the volume fractions of the hexagonal-close packed (HCP) phase prior to deformation were also considered. The results show that within the explored strain rate regime the TRIP-DP-HEA has a fairly low strain rate sensitivity parameter within the range from 0.004 to 0.04 , which is significantly lower than that of DP and TRIP steels. Samples with varying grain sizes (e.g., $\sim 2.8$ and $38 \mu \mathrm{m}$ ) and starting HCP phase fractions (e.g., $\sim 25$ and $72 \%$ ) at different strain rates show similar deformation mechanisms, i.e., dislocation plasticity and strain-induced transformation from the FCC matrix to the HCP phase. The low strain rate sensitivity is attributed to the observed dominant displacive transformation mechanism. Also, the coarse-grained alloy samples with a very high starting HCP phase fraction ( $72 \%$ ) prior to deformation show very good ductility with a total elongation of $\sim 60 \%$, suggesting that both, the initial and the transformed HCP phase in the TRIP-DP-HEA are ductile and deform further via dislocation slip at the different strain rates which were probed.

Keywords: high-entropy alloy, dual phase, phase transformation, strain rate sensitivity, grain size, deformation behavior

\section{INTRODUCTION}

Multi-component high-entropy alloys (HEAs) have shown continuous development of compositions, microstructures and desired properties over the last decade (Yeh et al., 2004; Cantor, 2014; Zhang et al., 2014; Li and Raabe, 2017b; Luo et al., 2017). Due to the inclusion of multiple principal elements, HEAs have much higher configurational entropy compared to traditional alloys and steels (Yeh et al., 2004). However, studies have also shown that the maximization of configurational entropy in equiatomic HEAs alone is not the decisive factor for the formation 
of stable single-phase solid solutions (Otto et al., 2013; Yao et al., 2014). This observation has motivated the design of novel non-equiatomic HEA systems with tunable phase stability (Li et al., 2016, 2017b; Li and Raabe, 2017b). One such alloy system is quaternary $\mathrm{Fe}_{80-x} \mathrm{Mn}_{x} \mathrm{Co}_{10} \mathrm{Cr}_{10}$ (at. \%), which shows the activation of displacive transformation mechanisms, e.g., twinning-induced plasticity (TWIP) and transformationinduced plasticity (TRIP) effects when decreasing the Mn content ( $x$-value) from 45 to 30 at. \% (Li et al., 2016; Li and Raabe, 2017b). Recently, a quinary system, i.e., $\mathrm{Co}_{20} \mathrm{Cr}_{20} \mathrm{Fe}_{40-y} \mathrm{Mn}_{20} \mathrm{Ni}_{y}$, showing also such tunable mechanisms when adjusting the $\mathrm{Ni}$ content ( $y$-value), has been developed (Li et al., 2017a).

Particularly, the quaternary $\mathrm{Fe}_{50} \mathrm{Mn}_{30} \mathrm{Co}_{10} \mathrm{Cr}_{10}$ and quinary $\mathrm{Co}_{20} \mathrm{Cr}_{20} \mathrm{Fe}_{34} \mathrm{Mn}_{20} \mathrm{Ni}_{6}$ HEAs in the above two systems show a dual-phase (DP) microstructure containing two compositionally equivalent high-entropy phases, i.e., the face-centered cubic (FCC) $\gamma$ and the hexagonal close-packed (HCP) $\varepsilon$ phases (Li et al., 2016, 2017a; Li and Raabe, 2017b; Wang et al., 2018). Due to the limited thermodynamic stability of the FCC matrix phase in these alloys, it undergoes a displacive phase transformation from FCC $\gamma$ into the HCP $\varepsilon$ phase upon mechanical loading. Hence, this type of non-equiatomic Dual-phase high-entropy alloys (DPHEA) concept combines the solid-solution strengthening effect inherent in HEAs with the TRIP effect known from advanced high-strength steels (Hadfield, 1888; Curtze et al., 2009; Raabe et al., 2013), leading to significantly improved strength and ductility compared to previous equiatomic single-phase HEAs ( $\mathrm{Li}$ et al., 2016, 2017a; Li and Raabe, 2017b; Nene et al., 2017).

As discussed above, the recently developed TRIP-assisted DPHEAs show comparable strengthening mechanisms and hence mechanical properties found also in many advanced steels, e.g., DP and TRIP steels (Hadfield, 1888; Curtze et al., 2009; Raabe et al., 2013). Many of these steels show positive and strong strain rate sensitivity upon deformation (Curtze et al., 2009). This feature has been attributed to the rationale that at higher strain rates the time for a dislocation to be rendered immobile in front of an obstacle (e.g., grain boundaries, twin boundaries and phase interfaces) before it can propagate upon gaining additional thermal energy is shorter than that at lower strain rates (Meyers, 1994; Curtze et al., 2009). For instance, high manganese containing TRIP/TWIP steels usually undergo deformation driven transformations involving formation of $\varepsilon$ and $\alpha$ martensite as well as mechanical twins (Grässel et al., 2000; Gutierrez-Urrutia and Raabe, 2011; Wong et al., 2016). The transformation paths could be affected by strain rate as the adiabatic heating modifies the phase transformation rate, leading to change in mechanical properties such as ultimate strength, uniform and total elongations (Sato et al., 1989; Grässel et al., 2000). Also, DP steels with a ferritic-martensitic microstructure (Fu et al., 2012; Tasan et al., 2015) are generally more sensitive to strain rate changes compared to TRIP steels consisting of bainite, martensite, retained austenite and ferrite matrix (Grässel et al., 2000; Huh et al., 2008).

Yet, the microstructure of the current DP HEA (FCC matrix plus HCP martensite) and the associated transformation pathways when exposed to loads (from the FCC matrix into HCP martensite) in the TRIP-DP-HEAs are essentially different from those in the above discussed DP and TRIP steels (Grässel et al., 2000; Li et al., 2017b; Niendorf et al., 2018; Wang et al., 2018). These differences may lead to different susceptibility to strain rate changes upon deformation. Motivated by this, the present work focuses on investigating the strain rate sensitivity of a typical TRIP-DP-HEA, i.e., quaternary $\mathrm{Fe}_{50} \mathrm{Mn}_{30} \mathrm{Co}_{10} \mathrm{Cr}_{10}$ (at. $\%)$, with varying starting FCC grain sizes and initially available HCP phase fractions at room temperature. The stress-strain behavior at various strain rates were studied and correlated to the microstructural evolution in the HEA samples.

\section{METHODOLOGY \\ Alloy Processing}

The TRIP-DP-HEA with a nominal composition of $\mathrm{Fe}_{50} \mathrm{Mn}_{30} \mathrm{Co}_{10} \mathrm{Cr}_{10}$ (at. \%) was synthesized from pure metals using vacuum induction furnace melting, followed by casting and hot-rolling at $900^{\circ} \mathrm{C}$ to $50 \%$ thickness reduction. After that the samples were homogenized at $1,200^{\circ} \mathrm{C}$ in Argon atmosphere for $2 \mathrm{~h}$ and then water quenched. The homogenized alloy plates were further cold-rolled to $60 \%$ thickness reduction and annealed at a furnace temperature of $900^{\circ} \mathrm{C}$ for $3 \mathrm{~min}$ in Argon atmosphere followed by water-quenching. The so produced fine-grained samples were used as one set of starting material.

Another set of coarse-grained samples with different HCP phase fraction was obtained by annealing the fine-grained samples further at $1,100^{\circ} \mathrm{C}$ for $2 \mathrm{~h}$ in argon atmosphere followed by water-quenching.

\section{Analysis}

Rectangular dog-bone shaped tensile specimens with gauge geometry of $4 \mathrm{~mm} \times 2 \mathrm{~mm} \times 1.5 \mathrm{~mm}$ were sectioned from the alloy sheets by using electrical discharge machining (EDM). Uniaxial tensile tests were performed using initial strain rate values of $5 \times 10^{-3} \mathrm{~s}^{-1}, 1 \times 10^{-3} \mathrm{~s}^{-1}, 5 \times 10^{-4} \mathrm{~s}^{-1}$, and $1 \times$ $10^{-4} \mathrm{~s}^{-1}$, respectively, at room temperature using a Kammrath and Weiss tensile stage. The strain rates were confined to this range to allow for coupling the tests with high resolution digital image correlation using the Aramis system from GOM GmbH (Li et al., 2017b). This approach enabled us to determine the local strain evolution with high accuracy, yet, at the cost of sacrificing some portion of the strain rate space. The local strain evolution probed during tensile deformation at each strain rate was then correlated to the microstructure evolution. Strain rate jump tests (Li et al., 2012) were also performed at room temperature using the same tensile stage with the above four strain rates. Three tensile specimens for each processing condition were tested to confirm reproducibility.

The un-deformed HEA samples with different starting grain sizes and the fractured tensile samples with different strain rates were metallographically prepared for microstructural characterization. The sample surfaces were first mechanically ground using $\mathrm{SiC}$ papers from 400 to 2,500 granulation, followed by polishing using $3 \mu \mathrm{m}$ diamond suspensions. Final polishing was performed using an oxide polishing suspension (OPS) with silica particle size of $\sim 50 \mathrm{~nm}$ to remove the deformation layer caused by mechanical grinding. After each step of polishing the 
samples were subjected to ultrasonic cleaning with ethanol before proceeding to next step.

Microstructural characterization was carried out using electron backscattered diffraction (EBSD) and electron channeling contrast imaging (ECCI). EBSD measurements were performed in a dual-beam Zeiss-crossbeam XB1560 scanning electron microscope (SEM) equipped with the TSL OIM data collection software. ECCI analysis was conducted using a Zeiss-Merlin instrument (Gutierrez-Urrutia et al., 2009). For the tensile tested samples, the deformation microstructures were analyzed via EBSD and ECCI at sample regions which had experienced different local strain levels. For the un-deformed samples, the compositional homogeneity was also analyzed by energy dispersive X-ray spectroscopy (EDS) using the Zeiss-Merlin instrument.

\section{RESULTS}

\section{Microstructure Prior to Deformation}

Figures 1a,b show the EBSD phase and inverse pole figure (IPF) maps of the fine-grained TRIP-DP-HEA, which reveal an average FCC matrix grain size of $\sim 2.8 \mu \mathrm{m}$ (excluding the twin boundaries) and an HCP martensite fraction of $\sim 25 \%$. The ECC images in Figures 1c,d show the dual-phase microstructure with a large number of stacking faults in the FCC matrix. These stacking faults as well as the HCP martensite were thermally induced during water-quenching (Li et al., 2016, 2017b).

Figure 2 shows a typical combined analysis of ECCI, EBSD, and EDS on the coarse-grained HEA sample which was obtained by annealing the above grain-refined sample at $1,100^{\circ} \mathrm{C}$ for $2 \mathrm{~h}$ followed by water-quenching. The ECC images (Figures 2a,b) and EBSD maps (Figures 2c,d) reveal distinct HCP martensitic laths in the FCC matrix. The HEA samples in this condition show an average FCC matrix grain size of $\sim 38 \mu \mathrm{m}$ and HCP phase fraction of $\sim 72 \%$. From the EDS maps in Figure 2e, the four principal elements, i.e., $\mathrm{Fe}, \mathrm{Mn}, \mathrm{Co}$, and $\mathrm{Cr}$ are uniformly distributed, confirming the compositional homogeneity of the alloy sample. Therefore, a potential influence of compositional inhomogeneity on the deformation mechanisms can be excluded (Li and Raabe, 2017a; Li et al., 2017c).

\section{Tensile Behavior and Fracture Morphologies at Varying Strain Rates}

Figure 3A displays the engineering stress-strain curves of the DPHEA samples with two different starting microstructures and at different strain rates (i.e., $5 \times 10^{-3} \mathrm{~s}^{-1}, 1 \times 10^{-3} \mathrm{~s}^{-1}, 5$ $\times 10^{-4} \mathrm{~s}^{-1}$, and $1 \times 10^{-4} \mathrm{~s}^{-1}$, respectively). The fine-grained DP-HEA samples with an average grain size of $\sim 2.8 \mu \mathrm{m}$ and a starting HCP phase fraction of $\sim 25 \%$ present very similar yield strength (YS, $\sim 300 \mathrm{MPa}$ ), ultimate tensile strength (UTS, $\sim 800$ $\mathrm{MPa})$ and total elongation $(\sim 60 \%)$ at the various strain rates. A similar trend is also observed for the coarse-grained DP-HEA samples with an average grain size of $\sim 38 \mu \mathrm{m}$ and a starting HCP phase fraction of $\sim 72 \%$. However, the coarse-grained DPHEA samples show much lower yield strength $(\sim 150 \mathrm{MPa})$ and ultimate tensile strength $(\sim 600 \mathrm{MPa})$ compared to the finegrained counterparts. This is mainly due to the Hall-Petch effect.
Interestingly, the coarse-grained DP-HEA samples with a very high HCP fraction $(\sim 72 \%)$ still show large uniform elongation ( $\sim 60 \%)$. This observation suggests that the HCP phase formed in the DP-HEA is also ductile.

Figure 3B shows the strain hardening response of the two types of DP-HEA samples at varying strain rates. The strain hardening rates of the DP-HEA samples are also very similar at different strain rates though the hardening rates of the finegrained $(\sim 2.8 \mu \mathrm{m})$ samples are slightly higher than those of the coarse-grained $(\sim 38 \mu \mathrm{m})$ materials.

As shown above, from the constant strain rate tensile tests, the differences in the strength values between the coarse and finegrained DP-HEA samples at different strain rates are too small to be used for calculation of the strain rate sensitivity parameter $(m)$. To further confirm the insignificant strain rate effects on the deformation behavior of the DP-HEA, a set of fine-grained samples were used to perform strain rate jump tests, in which samples were subjected to rapid changes of strain rates (i.e., $5 \times$ $10^{-3} \mathrm{~s}^{-1}, 1 \times 10^{-3} \mathrm{~s}^{-1}, 5 \times 10^{-4} \mathrm{~s}^{-1}$, and $1 \times 10^{-4} \mathrm{~s}^{-1}$ ) without intermediate unloading. Figures $4 \mathbf{A}, \mathbf{B}$ show the corresponding stress-strain curves of the fine-grained DP-HEA samples with increasing and decreasing strain rates, respectively. The strain rate sensitivity parameters $(m)$ for each jump of the effective strain rate were calculated by the following equation:

$$
m=\frac{\log \left(\frac{\sigma_{x}}{\sigma_{x+1}}\right)}{\log \left(\frac{\dot{\epsilon}_{x}}{\dot{\epsilon}_{x+1}}\right)}
$$

where $\sigma_{x}$ and $\dot{\epsilon}_{x}$ are the flow stress and strain rate before the jump, respectively; $\sigma_{x+1}$ and $\dot{\epsilon}_{x+1}$ are the flow stress and strain rate after the jump, respectively. As shown in Figure 4, the calculated strain rate sensitivity for increasing strain rate tensile tests ranges between 0.013 and 0.004 , and that for decreasing strain rate tensile tests between 0.007 and 0.038 . These very small values suggest that the change in strain rate has very little or negligible effect on the stress-strain behavior of the DP-HEA samples, which is also consistent with the results from constant strain rate tensile tests.

Figure 5 shows the fracture morphologies of the DP-HEA samples with varying grain sizes and initially available HCP phase fractions at different strain rates after the constant strain rate tensile tests. It is clearly indicated that all the samples were fractured in a ductile way which is characterized by well-defined dimples. The size of dimples formed on the fracture surfaces of the coarse-grained samples with average grain size of $\sim 38 \mu \mathrm{m}$ (Figures 5a-d) are larger than those for the fine-grained samples with average grain size of $2.8 \mu \mathrm{m}$ (Figures $\mathbf{5 e - h}$ ), which reflects the grain size effect on the fracture behavior.

\section{Microstructural Evolution Upon Deformation}

Figures 6a-c show the EBSD phase maps of the fine-grained $(\sim 2.8 \mu \mathrm{m})$ DP-HEA sample after tensile testing at a constant strain rate of $1 \times 10^{-4} \mathrm{~s}^{-1}$. With the increase of local strain from 30 to $50 \%$, and further to $80 \%$, the HCP phase fraction increases from 42 to $60 \%$, and further to $67 \%$, respectively. The ECC 

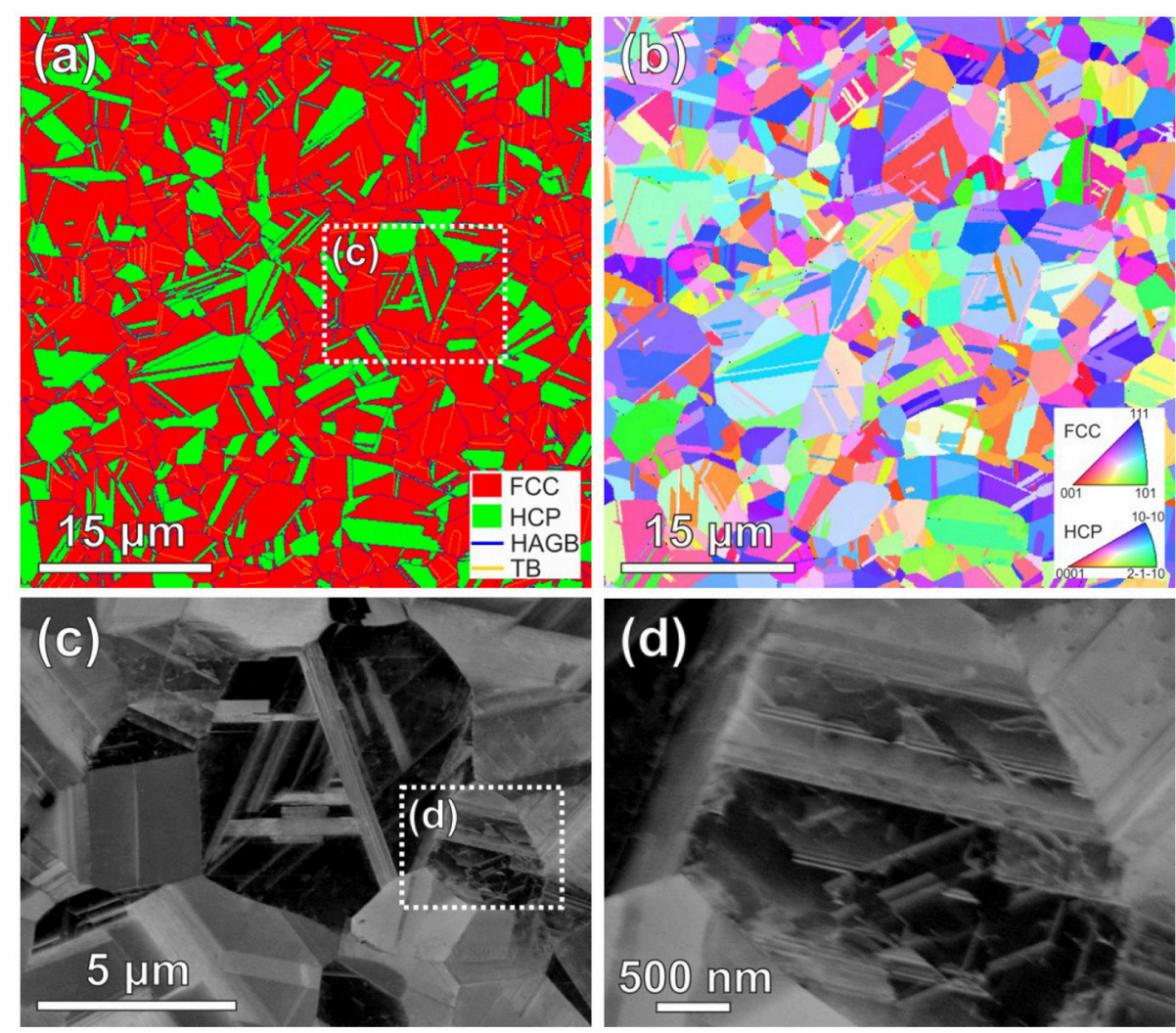

FIGURE 1 | Combined EBSD and ECCl analysis on the fine-grained dual-phase HEA prior to deformation: (a) EBSD phase and boundary map; (b) EBSD IPF map of an identical sample region in (a); (c) ECC image of the marked region in (a); (d) ECC image of the marked region in (c) with relatively high magnification showing stacking faults in the FCC matrix.

images taken from the marked regions in Figures 6a-c are shown in Figures 6d-f, respectively, and they indicate the lath-shaped HCP martensite formed during the tensile loading.

Figure 7 shows the deformation microstructures corresponding to varying local strains in the fine-grained $(\sim 2.8 \mu \mathrm{m})$ alloy sample after tensile testing at constant strain rate of $5 \times 10^{-3} \mathrm{~s}^{-1}$. Analogous to the sample tested at lower strain rate as shown above, the HCP phase fraction in the fine-grained DP-HEA samples at higher strain rate also increases gradually with increasing local strain levels. As the local strain increases, the sample region with an EBSD confidence index below 0.1 (i.e., black region in the phase maps) also increases owing to the high amount of deformation that distorts the FCC and HCP lattice beyond the resolution limits as required for reliable indexing by EBSD. From the ECC images (Figures 7d-f), the HCP phase formed at the early stage of deformation has a well-defined lath-shape (Figure 7d) which also undergoes significant deformation at the later stages of tensile deformation (Figure 7f).

Figure 8 shows the deformation microstructure in the coarsegrained $(\sim 38 \mu \mathrm{m})$ DP-HEA sample with a starting HCP phase fraction of $\sim 72 \%$ upon tensile testing at a constant strain rate of $1 \times 10^{-4} \mathrm{~s}^{-1}$. Irrespective of the high starting fraction of $\mathrm{HCP}$ phase prior to loading, the microstructure still undergoes further deformation induced transformation from the remaining FCC phase into the HCP martensite. At the sample region near to the fracture surface (i.e., with a local strain of $\sim 80 \%$ ), the FCC phase fraction reduces to as little as only $\sim 3 \%$ (Figure 8c). Similarly, Figure 9 presents the deformation microstructures at various local strain levels $(30,50$, and $80 \%)$ in the coarsegrained $(\sim 38 \mu \mathrm{m})$ DP-HEA sample with a starting HCP phase fraction of $\sim 72 \%$ upon tensile testing at a constant strain rate of $5 \times 10^{-3} \mathrm{~s}^{-1}$. At the region with a local strain of $\sim 80 \%$ (near to the fracture surface) in the coarse-grained DP-HEA sample, the remaining FCC phase fraction is $~ 5 \%$ (Figure 9c), similar to that in the sample deformed at a lower strain rate of $1 \times 10^{-4} \mathrm{~s}^{-1}(\sim 3 \%$; Figure $8 \mathrm{c})$. The substructures including slip bands in the HCP martensite in the deformed sample are documented by the ECC images shown in Figures 9d-f, which are consistent with previous investigations on the deformation microstructures of the DP-HEAs (Li et al., 2016).

\section{DISCUSSION}

The TRIP-assisted DP-HEA investigated in the present study exhibits strengthening mechanisms similar to those in some 

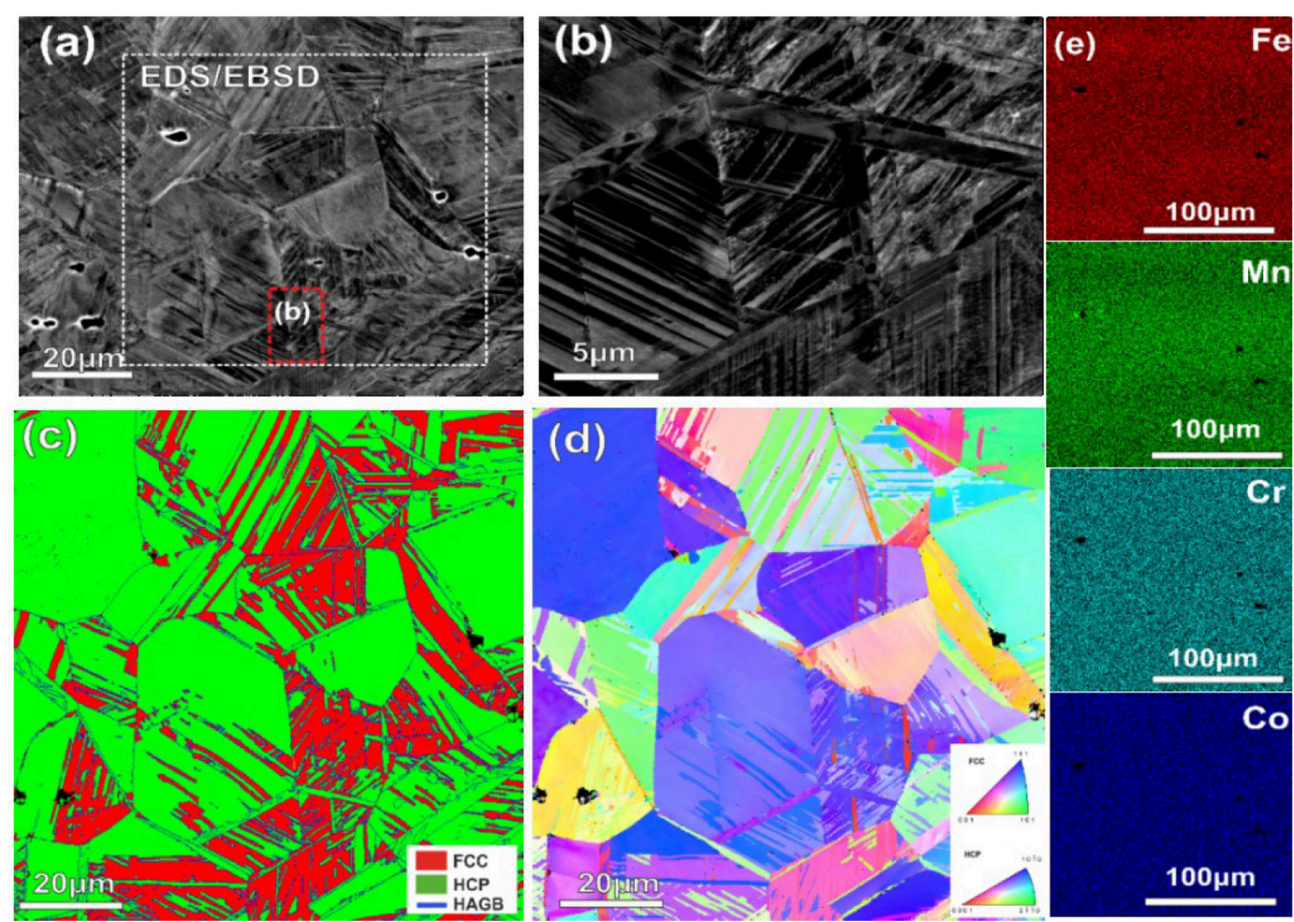

FIGURE 2 | Combined ECCI, EBSD and EDS analysis on the coarse-grained dual-phase HEA prior to deformation: (a) ECC image with relatively low magnification; (b) ECC image of the smaller marked region in (a) showing the martensite laths; (c) EBSD phase and boundary map of the larger marked region in (a); (d) EBSD IPF map of the identical region in (c); (e) EDS maps of the larger region marked in (a) showing the distribution of the four principal elements, i.e., Fe, Mn, Co, and Cr.
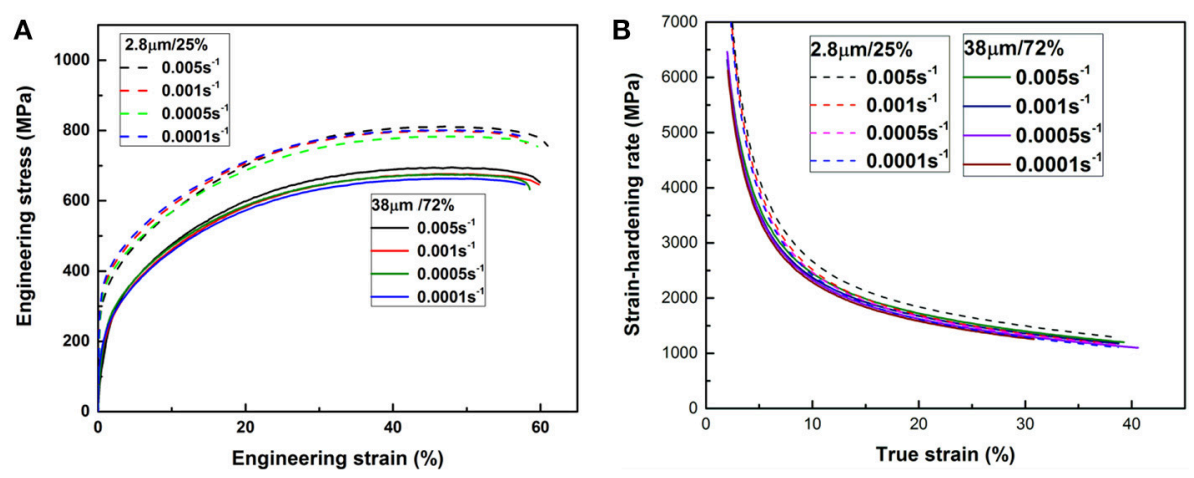

FIGURE 3 | (A) Typical tensile stress-strain curves of the DP-HEAs with varying FCC grain sizes and initially available HCP phase fractions. (B) Strain hardening curves with respect to true strain corresponding to the stress-strain curves shown in (A).

advanced steels, e.g., DP and TRIP steels (Hadfield, 1888; Curtze et al., 2009; Raabe et al., 2013). Many of these materials show high sensitivity on strain rate changes during deformation. For instance, the strain rate sensitivity parameters for the DP 600 and TRIP 700 steels have been reported to be 18.5 and 11.3, respectively, as probed over a wide strain rate range from $10^{-3}$ to $1250 \mathrm{~s}^{-1}$ (Curtze et al., 2009). The relatively high strain rate sensitivity of these materials is due to the fact that at higher strain rates the time over which a dislocation is immobile, requiring additional thermal energy to overcome obstacles (e.g., grain boundaries, twin boundaries, and phase interfaces), to continue propagation is shorter than that at lower strain rates (Meyers, 1994; Curtze et al., 2009). However, it is observed that the mechanical properties of the TRIP-assisted DP-HEA samples in the present study are not obviously influenced by the change in strain rate based on the very low strain rate sensitivity $(<0.04)$. Although the strain rates probed in the current study are confined to the range from $1 \times 10^{-4}$ to $0.5 \times 10^{-2} \mathrm{~s}^{-1}$ due to the limits set by the DIC-assisted tensile testing protocol, similar strain rate ranges have also been applied in previous works to achieve reliable strain rate sensitivity for other materials, e.g., Inconel 718 alloy (Urdanpilleta et al., 2005). Therefore, the results from the 

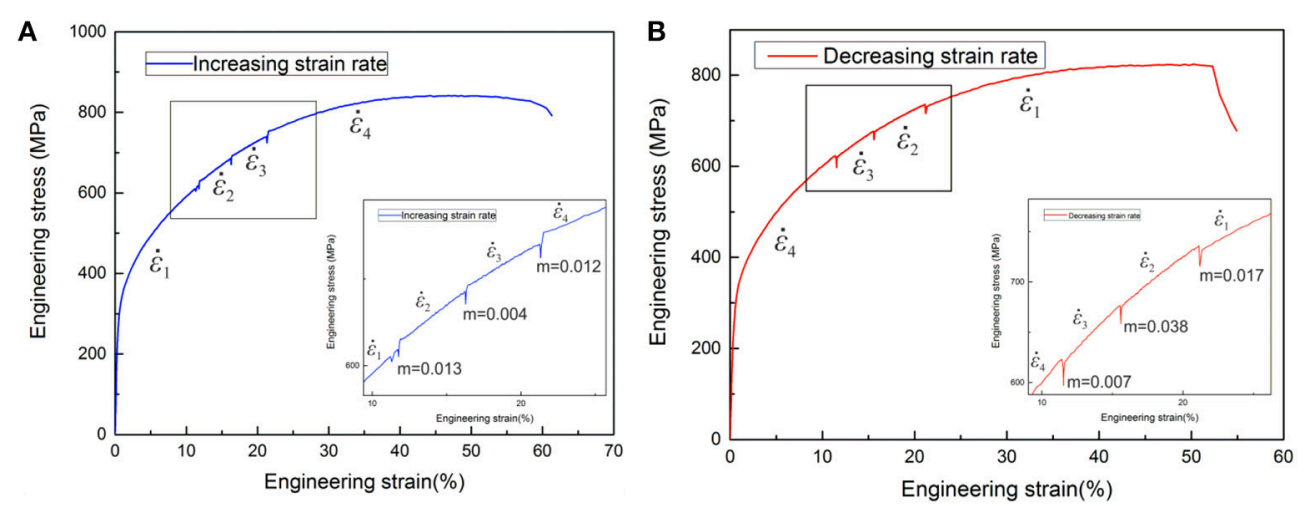

FIGURE 4 | Typical stress-strain curves obtained from the strain rate jump tensile tests of the fine-grained DP-HEA samples. (A) Curves with increasing strain rate; (B) Curves with decreasing strain rate. The values of strain rate sensitivity $(m)$ for each jump of the effective strain rate are denoted at the nodes in the inserts.
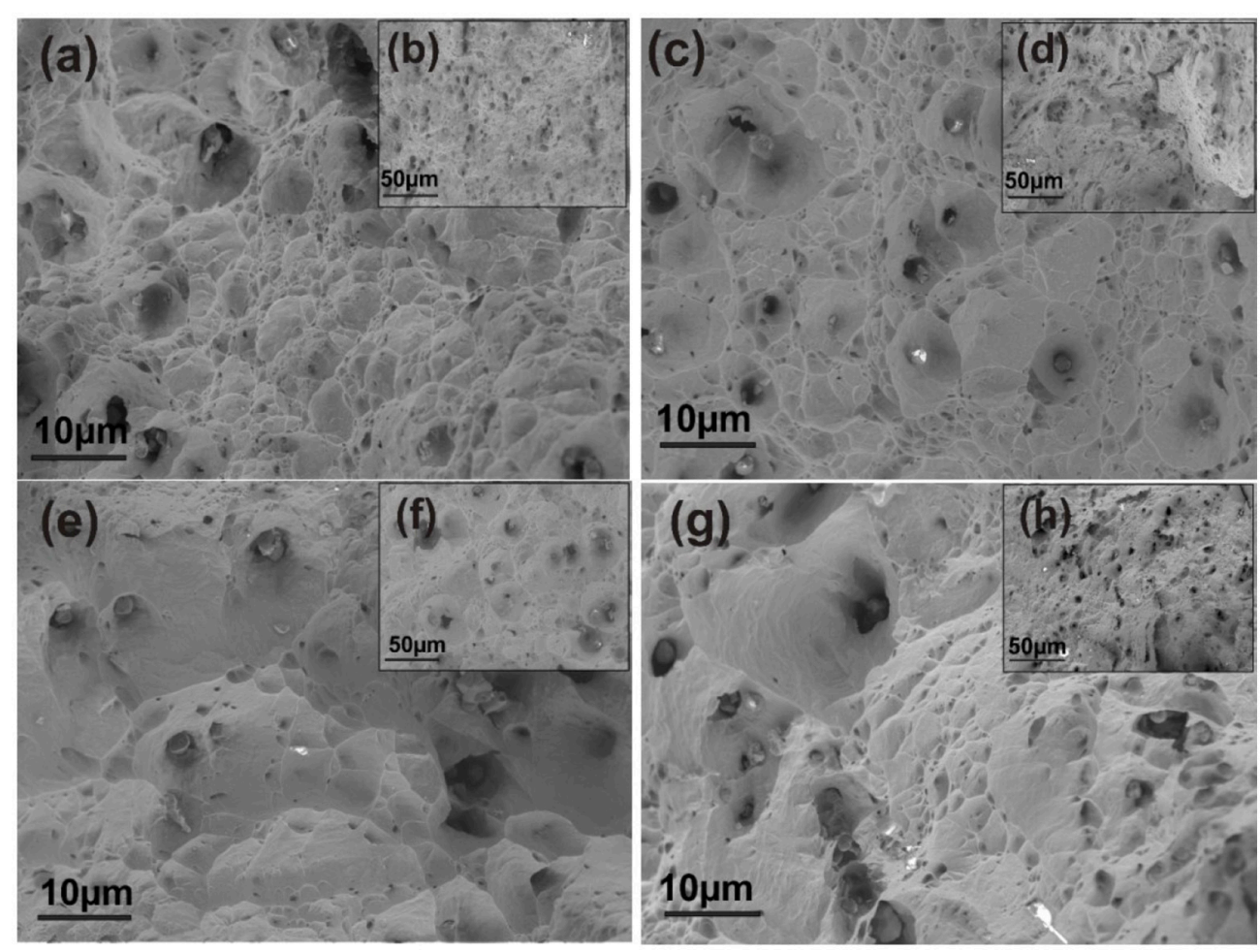

FIGURE 5 | Fracture morphologies of the dual-phase HEA samples with varying grain sizes at different strain rates after the constant strain rate tensile tests. (a,b) Fine-grained and constant strain rate of $1 \times 10^{-4} \mathrm{~s}^{-1}$; (c,d) Fine-grained and constant strain rate of $5 \times 10^{-3} \mathrm{~s}^{-1}$; (e,f) Coarse-grained and constant strain rate of $1 \times 10^{-4} \mathrm{~s}^{-1} ; \mathbf{( g , h}$ ) Coarse-grained and constant strain rate of $5 \times 10^{-3} \mathrm{~s}^{-1}$.

current study are considered to be adequate to reflect a very low strain rate sensitivity of the TRIP-assisted DP-HEAs.

Since the displacive transformation from the FCC matrix to the HCP martensite is-besides dislocation glide-the predominant mechanism during deformation of the DPHEA, the very low sensitivity to strain rate changes of the DP-HEA can be mainly ascribed to the athermal nature of the displacive transformation the kinetics of which is rather insensitive to strain rate changes in the range from
$1 \times 10^{-4} \mathrm{~s}^{-1}$ to $0.5 \times 10^{-2}$ as observed in the current study. Further we find that the differences in adiabatic heating associated with the different strain rates during deformation do not obviously affect the phase transformation process.

According to the microstructural observations after tensile testing, two trends can be established regarding the phase transformation process upon deformation in the two different DP-HEA samples at different strain rates. First, in samples with 

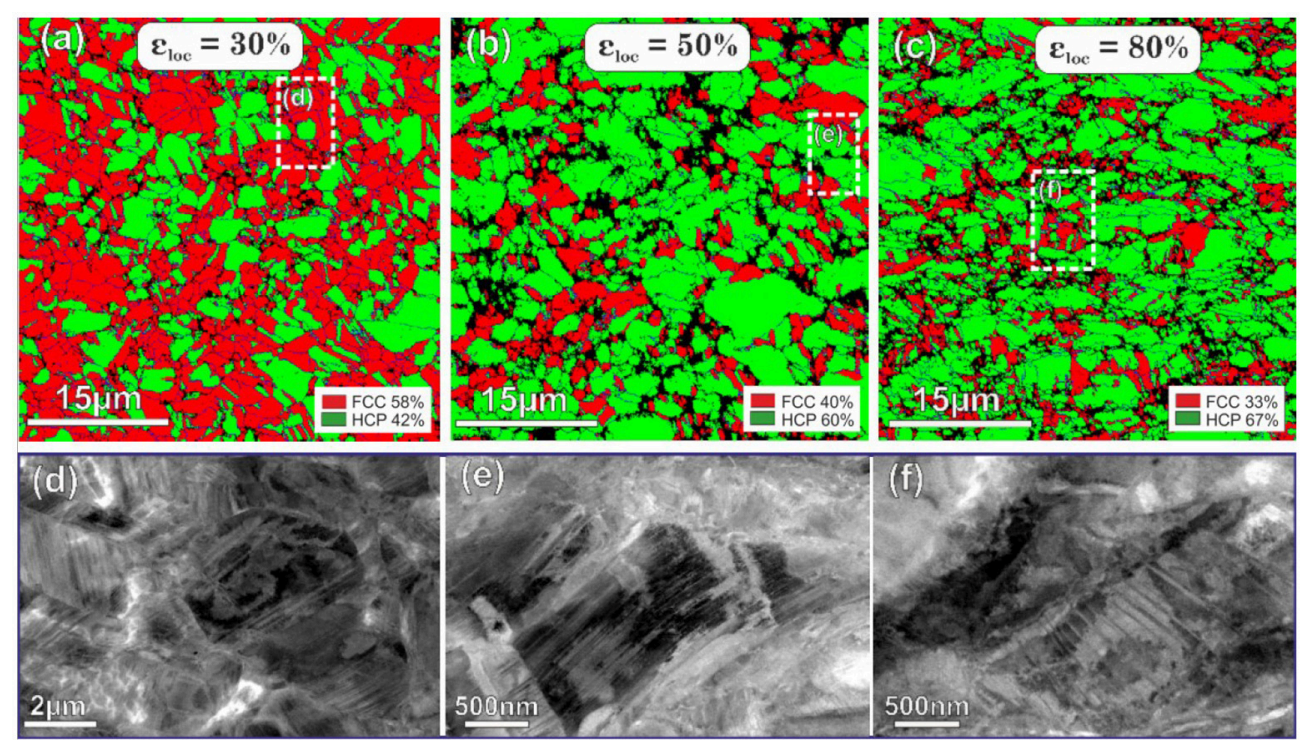

FIGURE 6 | EBSD phase maps (a-c) and ECC images (d-f) showing the deformation microstructures in the fine-grained DP-HEA sample with an average grain size of $\sim 2.8 \mu \mathrm{m}$ and a starting HCP phase fraction of $\sim 25 \%$ at varying local strain $\left(\varepsilon_{\text {loc }}\right)$ levels $(30,50$, and $80 \%)$ after tensile testing at a constant strain rate of $1 \times 10^{-4} \mathrm{~s}^{-1}$.

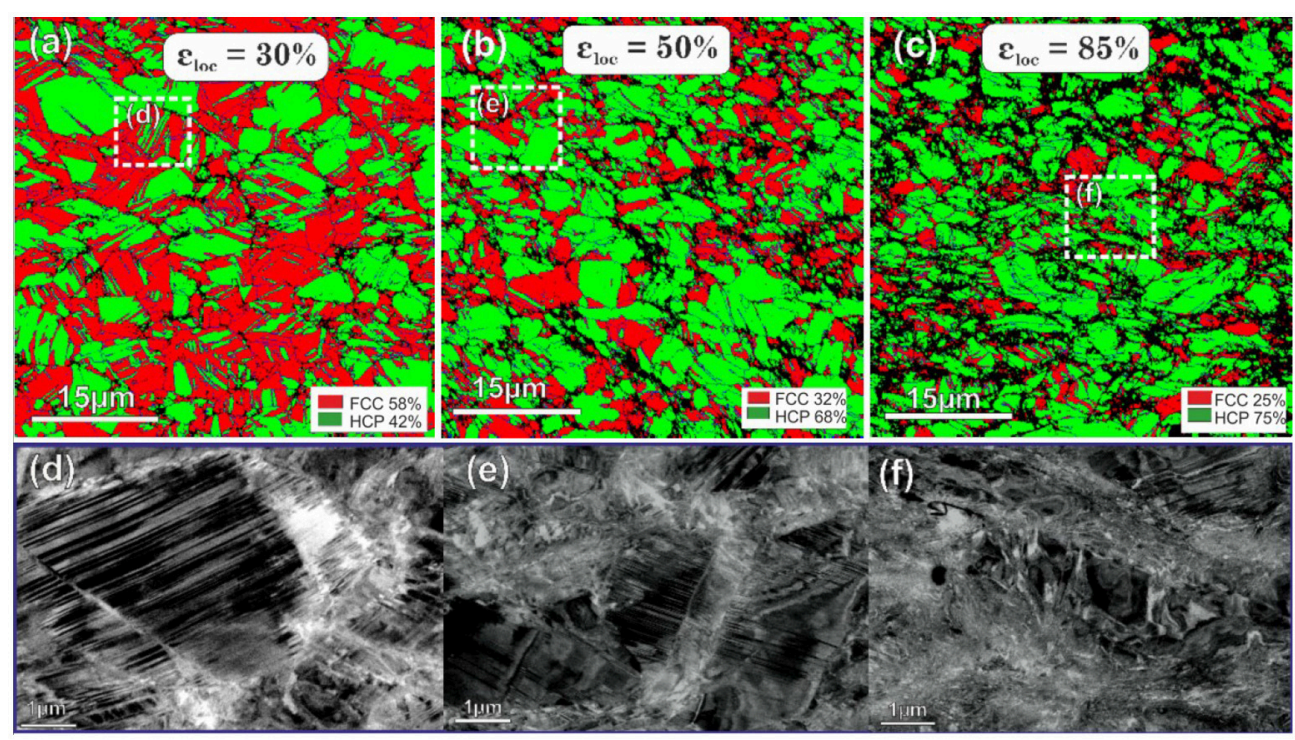

FIGURE 7 | EBSD phase maps (a-c) and ECC images (d-f) showing the deformation microstructures in the fine-grained DP-HEA sample with an average grain size of $\sim 2.8 \mu \mathrm{m}$ and a starting HCP phase fraction of $\sim 25 \%$ at varying local strain $\left(\varepsilon_{\text {loc }}\right)$ levels $(30,50$ and $85 \%)$ after tensile testing at a constant strain rate of $5 \times 10^{-3} \mathrm{~s}^{-1}$.

an identical starting microstructure (i.e., the same average grain size and HCP phase fraction), the transformation process was carried on in a similar way and with a similar amount of transformed FCC phase after tensile deformation at different strain rates. This indicates that the change in the strain rate does not significantly alter the kinetics of the dynamic phase transformation. Second, the fraction of FCC phase transformed to HCP martensite in the coarse-grained sample ( $\sim 24 \%$; Figure 8) is much lower than that in the fine-grained counterpart
( $\sim 42 \%$; Figure 6) after tensile loading up to a local strain of $\sim 80 \%$. However, the coarse-grained sample also shows good ductility comparable to that of the fine-grained material. This observation suggests that the HCP martensite in the coarsegrained DP-HEA sample is in itself also sufficiently ductile to accommodate a large amount of strain at all the strain rates which were probed. This can be explained by our previous work which showed that the HCP martensite phase deforms via multiple deformation mechanisms including dislocation slip, 


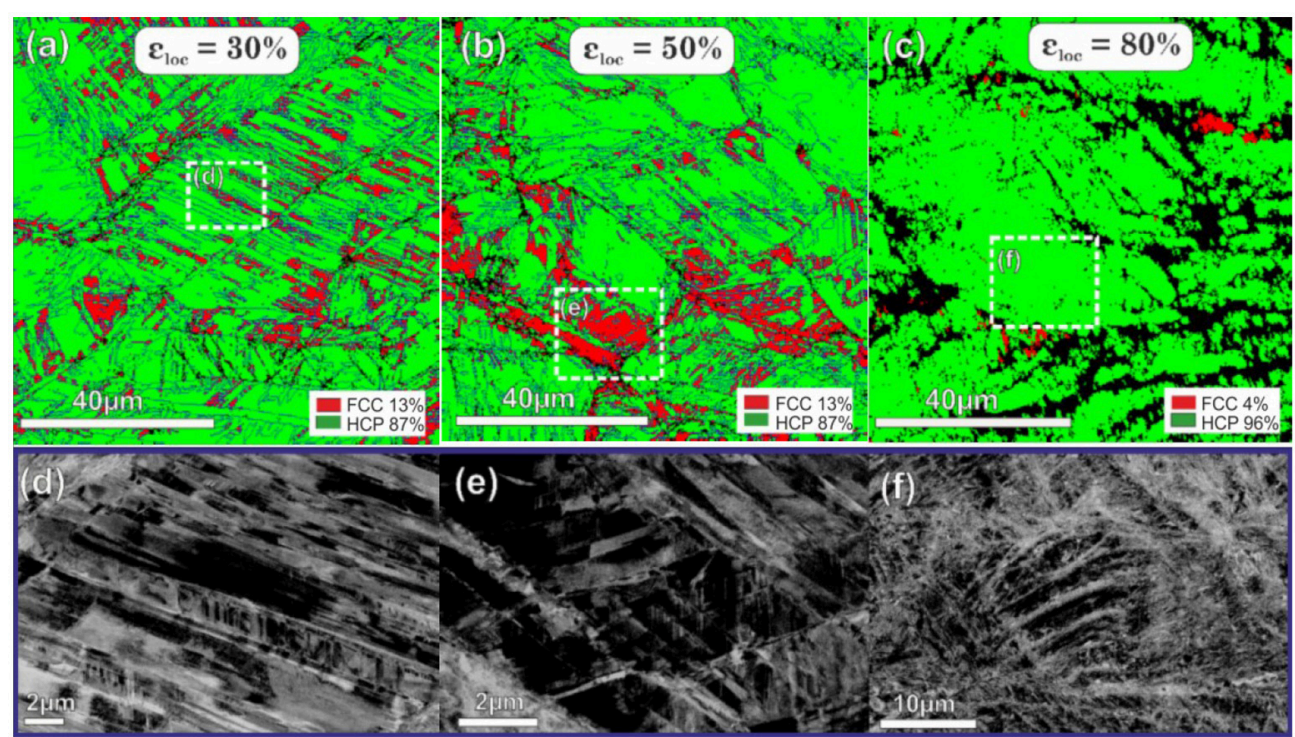

FIGURE 8 | EBSD phase maps (a-c) and ECC images (d-f) showing the microstructures in the coarse-grained DP-HEA sample with an average grain size of $\sim 38 \mu \mathrm{m}$ and a starting $\mathrm{HCP}$ phase fraction of $\sim 72 \%$ at varying local strain $\left(\varepsilon_{\text {loc }}\right)$ levels $(30,50$, and $80 \%)$ after tensile testing at a constant strain rate of $1 \times 10^{-4} \mathrm{~s}^{-1}$.
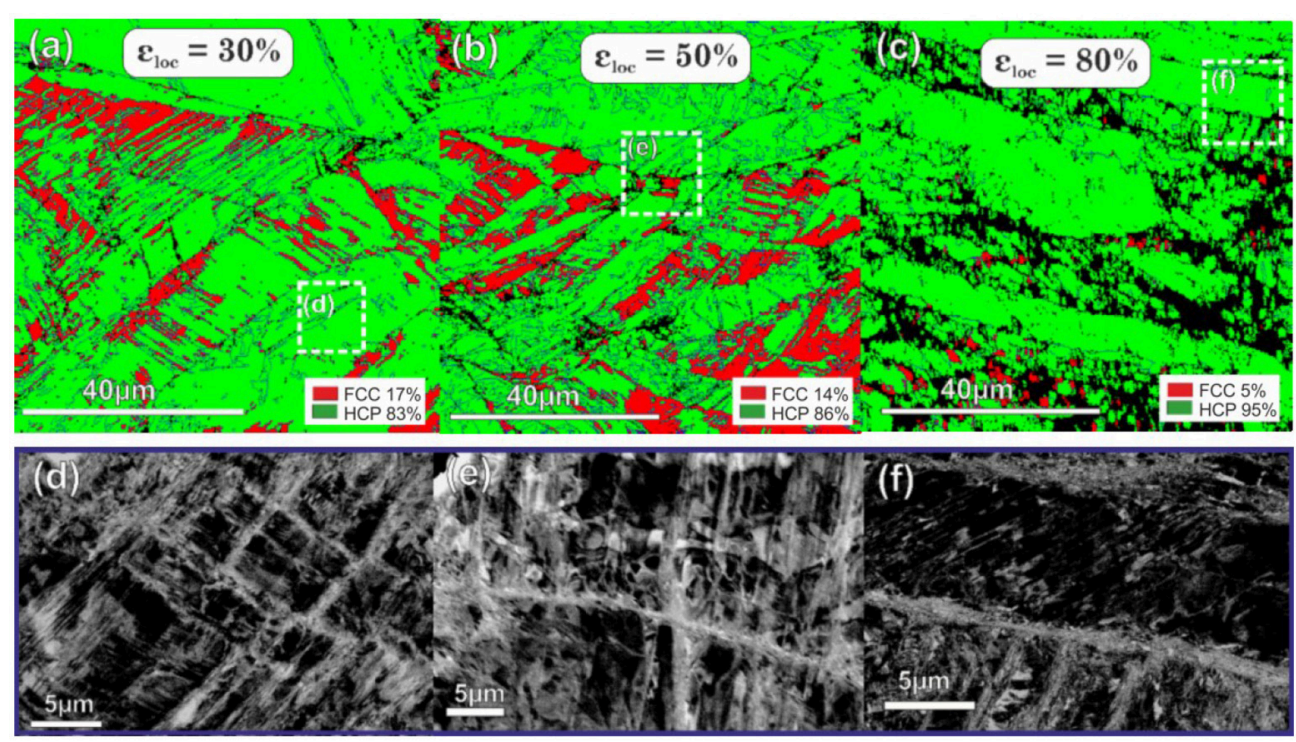

FIGURE $\mathbf{9}$ | EBSD phase maps (a-c) and ECC images (d-f) showing the microstructures in the coarse-grained DP-HEA sample with an average grain size of $\sim 38 \mu \mathrm{m}$ and a starting HCP phase fraction of $\sim 72 \%$ at varying local strain $\left(\varepsilon_{\text {loc }}\right)$ levels $(30,50$, and $80 \%)$ after tensile testing at a constant strain rate of $5 \times 10^{-3} \mathrm{~s}^{-1}$.

formation of stacking faults and mechanical twinning (Li et al., 2016, 2017b).

\section{CONCLUSIONS}

We investigated the strain rate sensitivity of a typical TRIPassisted dual-phase HEA, i.e., quaternary $\mathrm{Fe}_{50} \mathrm{Mn}_{30} \mathrm{Co}_{10} \mathrm{Cr}_{10}$ (at. $\%)$, for different grain sizes and initially available HCP phase fractions at room temperature. The stress-strain behavior as well as the microstructural evolution at various strain rates (i.e., $5 \times$ $10^{-3} \mathrm{~s}^{-1}, 1 \times 10^{-3} \mathrm{~s}^{-1}, 5 \times 10^{-4} \mathrm{~s}^{-1}$, and $1 \times 10^{-4} \mathrm{~s}^{-1}$ ) has been probed. The following conclusions are made:

(1) The strain rate sensitivity $(m)$ of the TRIP-assisted dualphase HEA with two different starting microstructures at the probed strain rates is fairly low with the values within the range of 0.004-0.04, suggesting that the alloy can withstand the change in applied strain rate during forming and fabrication. 
(2) Strain-induced displacive transformation from the FCC matrix to the HCP martensite is the primary deformation mechanism-besides dislocation glide-for both of the fine and coarse-grained dual-phase HEA samples studied under different strain rates at room temperature.

(3) The low strain rate sensitivity of the TRIP-assisted dualphase HEAs is attributed to the prevalent displacive and athermal transformation mechanism acting during deformation.

(4) DP-HEA samples with very high starting HCP phase fraction can also have good ductility at different strain rates, suggesting that the HCP phase is also ductile, deforming further by dislocation glide and twinning.

\section{REFERENCES}

Cantor, B. (2014). Multicomponent and high entropy alloys. Entropy 16:4749. doi: 10.3390/e16094749

Curtze, S., Kuokkala, V. T., Hokka, M., and Peura, P. (2009). Deformation behavior of TRIP and DP steels in tension at different temperatures over a wide range of strain rates. Mater. Sci. Eng. A 507, 124-131. doi: 10.1016/j.msea.2008. 11.050

Fu, L., Li, Z., Wang, H., Wang, W., and Shan, A. (2012). Lüders-like deformation induced by delta-ferrite-assisted martensitic transformation in a dual-phase high-manganese steel. Scr. Mater. 67, 297-300. doi: 10.1016/j.scriptamat.2012.05.010

Grässel, O., Krüger, L., Frommeyer, G., and Meyer, L. (2000). High strength $\mathrm{Fe}-\mathrm{Mn}-(\mathrm{Al}, \mathrm{Si})$ TRIP/TWIP steels development-propertiesapplication. Int. J. Plast. 16, 1391-1409. doi: 10.1016/S0749-6419(00)0 0015-2

Gutierrez-Urrutia, I., and Raabe, D. (2011). Dislocation and twin substructure evolution during strain hardening of an Fe-22 wt.\% Mn-0.6 wt.\% C TWIP steel observed by electron channeling contrast imaging. Acta Mater. 59, 6449-6462. doi: 10.1016/j.actamat.2011.07.009

Gutierrez-Urrutia, I., Zaefferer, S., and Raabe, D. (2009). Electron channeling contrast imaging of twins and dislocations in twinning-induced plasticity steels under controlled diffraction conditions in a scanning electron microscope. Scr. Mater. 61, 737-740. doi: 10.1016/j.scriptamat.2009. 06.018

Hadfield, R. A. (1888). Hadfield's manganese steel. Science 12, 284-286. doi: $10.1126 /$ science.ns-12.306.284

Huh, H., Kim, S.-B., Song, J.-H., and Lim, J.-H. (2008). Dynamic tensile characteristics of TRIP-type and DP-type steel sheets for an auto-body. Int. J. Mech. Sci. 50, 918-931. doi: 10.1016/j.ijmecsci.2007.09.004

Li, Z., Fu, L., Fu, B., and Shan, A. (2012). Effects of annealing on microstructure and mechanical properties of nano-grained titanium produced by combination of asymmetric and symmetric rolling. Mater. Sci. Eng. A 558, 309-318. doi: 10.1016/j.msea.2012.08.005

Li, Z., Körmann, F., Grabowski, B., Neugebauer, J., and Raabe, D. (2017a). $\mathrm{Ab}$ initio assisted design of quinary dual-phase high-entropy alloys with transformation-induced plasticity. Acta Mater. 136, 262-270. doi: 10.1016/j.actamat.2017.07.023

Li, Z., Pradeep, K. G., Deng, Y., Raabe, D., and Tasan, C. C. (2016). Metastable high-entropy dual-phase alloys overcome the strengthductility trade-off. Nature 534, 227-230. doi: 10.1038/nature 17981

Li, Z., and Raabe, D. (2017a). Influence of compositional inhomogeneity on mechanical behavior of an interstitial dual-phase high-entropy alloy. Mater. Chem. Phys. 210, 29-36. doi: 10.1016/j.matchemphys.2017. 04.050

Li, Z., and Raabe, D. (2017b). Strong and ductile non-equiatomic high-entropy alloys: design, processing, microstructure, and mechanical properties. JOM 69, 2099-2106. doi: 10.1007/s11837-017-2540-2

\section{AUTHOR CONTRIBUTIONS}

SB performed the experiments; ZL, KP, and DR supervised the project; SB and ZL analyzed the data; SB and ZL wrote the paper. All authors commented on the manuscript.

\section{ACKNOWLEDGMENTS}

The authors would like to gratefully acknowledge the kind support of M. Nellessen, M. Adamek, B. Breitbach, and F. Schlüter at the Max-Planck-lnstitut für Eisenforschung. The author (SB) would also like to thank the financial support from the German Academic Exchange Service (DAAD).

Li, Z., Tasan, C. C., Pradeep, K. G., and Raabe, D. (2017b). A TRIP-assisted dual-phase high-entropy alloy: grain size and phase fraction effects on deformation behavior. Acta Mater. 131, 323-335. doi: 10.1016/j.actamat.2017. 03.069

Li, Z., Tasan, C. C., Springer, H., Gault, B., and Raabe, D. (2017c). Interstitial atoms enable joint twinning and transformation induced plasticity in strong and ductile high-entropy alloys. Sci. Rep. 7:40704. doi: 10.1038/srep 40704

Luo, H., Li, Z., and Raabe, D. (2017). Hydrogen enhances strength and ductility of an equiatomic high-entropy alloy. Sci. Rep. 7:9892. doi: 10.1038/s41598-017-10774-4

Meyers, M. A. (1994). Dynamic Behavior of Materials. John wiley \& sons.

Nene, S. S., Liu, K., Frank, M., Mishra, R. S., Brennan, R. E., Cho, K. C., et al. (2017). Enhanced strength and ductility in a friction stir processing engineered dual phase high entropy alloy. Sci. Rep. 7:16167. doi: 10.1038/s41598-017-1 $6509-9$

Niendorf, T., Wegener, T., Li, Z., and Raabe, D. (2018). Unexpected cyclic stress-strain response of dual-phase high-entropy alloys induced by partial reversibility of deformation. Scr. Mater. 143, 63-67. doi: 10.1016/j.scriptamat.2017.09.013

Otto, F., Yang, Y., Bei, H., and George, E. P. (2013). Relative effects of enthalpy and entropy on the phase stability of equiatomic highentropy alloys. Acta Mater. 61, 2628-2638. doi: 10.1016/j.actamat.2013. 01.042

Raabe, D., Sandlöbes, S., Millán, J., Ponge, D., Assadi, H., Herbig, M., et al. (2013). Segregation engineering enables nanoscale martensite to austenite phase transformation at grain boundaries: a pathway to ductile martensite. Acta Mater. 61, 6132-6152. doi: 10.1016/j.actamat.2013. 06.055

Sato, K., Ichinose, M., Hirotsu, Y., and Inoue, Y. (1989). Effects of deformation induced phase transformation and twinning on the mechanical properties of austenitic Fe-Mn-Al alloys. ISIJ Int. 29, 868-877. doi: 10.2355/isijinternational.29.868

Tasan, C. C., Diehl, M., Yan, D., Bechtold, M., Roters, F., Schemmann, L., et al. (2015). An overview of dual-phase steels: advances in microstructureoriented processing and micromechanically guided design. Аnnu. Rev. Mater. Sci. 45, 391-431. doi: 10.1146/annurev-matsci-070214-0 21103

Urdanpilleta, M., Martínez-Esnaola, J. M., and Sevillano, J. G. (2005). Strain rate sensitivity of superplastic Inconel 718. Mater. Trans. 46, 1711-1719. doi: 10.2320/matertrans.46.1711

Wang, M., Li, Z., and Raabe, D. (2018). In-situ SEM observation of phase transformation and twinning mechanisms in an interstitial highentropy alloy. Acta Mater. 147, 236-246. doi: 10.1016/j.actamat.2018. 01.036

Wong, S. L., Madivala, M., Prahl, U., Roters, F., and Raabe, D. (2016). A crystal plasticity model for twinning-and transformation-induced plasticity. Acta Mater. 118, 140-151. doi: 10.1016/j.actamat.2016. 07.032 
Yao, M. J., Pradeep, K. G., Tasan, C. C., and Raabe, D. (2014). A novel, single phase, non-equiatomic FeMnNiCoCr high-entropy alloy with exceptional phase stability and tensile ductility. Scr. Mater. 72-73, 5-8. doi: 10.1016/j.scriptamat.2013.09.030

Yeh, J. W., Chen, S. K., Lin, S. J., Gan, J. Y., Chin, T. S., Shun, T. T., et al. (2004). Nanostructured high-entropy alloys with multiple principal elements: novel alloy design concepts and outcomes. Adv. Eng. Mater. 6, 299-303. doi: 10.1002/adem.200300567

Zhang, Y., Zuo, T. T., Tang, Z., Gao, M. C., Dahmen, K. A., Liaw, P. K., et al. (2014). Microstructures and properties of high-entropy alloys. Prog. Mater. Sci. 61, 1-93. doi: 10.1016/j.pmatsci.2013.10.001
Conflict of Interest Statement: The authors declare that the research was conducted in the absence of any commercial or financial relationships that could be construed as a potential conflict of interest.

Copyright (c) 2018 Basu, Li, Pradeep and Raabe. This is an open-access article distributed under the terms of the Creative Commons Attribution License (CC $B Y)$. The use, distribution or reproduction in other forums is permitted, provided the original author(s) and the copyright owner are credited and that the original publication in this journal is cited, in accordance with accepted academic practice. No use, distribution or reproduction is permitted which does not comply with these terms. 